\title{
Time-Dependent Changes in Urinary Metabolome Before and After Intensive Phase Tuberculosis Therapy: A Pharmacometabolomics Study
}

\author{
Monique Combrink, ${ }^{1}$ Ilse du Preez, ${ }^{1}$ Katharina Ronacher, ${ }^{2,3}$ Gerhard Walzl, ${ }^{3}$ and Du Toit Loots ${ }^{1}$
}

\begin{abstract}
Pharmacometabolomics is a rapidly emerging omics science signaling the convergence of clinical pharmacology, metabolomics, precision medicine, and biomarker research. Tuberculosis (TB) treatment outcomes have complex biological, environmental, and social determinants and thus, represent a promising application of pharmacometabolomics. In samples of 23 patients undergoing intensive phase TB therapy for 4 weeks, we identified drug-induced host-metabolome variations before and at repeated time intervals post-treatment: (1) an overall reduction in the oxidative stress levels over the course of TB treatment; (2) a time-dependent induction and inhibition of several enzymes in response to the drugs (CYP2E1, CYP3A4, alcohol dehydrogenase, and aminocarboxymuconate-semialdehyde decarboxylase), and altered oxidative stress levels (aconitase, formylglycinegenerating enzyme, $\alpha$-ketoglutarate dehydrogenase, and succinate-semialdehyde dehydrogenase); (3) an upregulated urea cycle; and (4) altered insulin production. This is the first study of its kind to indicate changes to the host metabolome in response to intensive TB treatment, at different time intervals during the course of treatment. These results provide new insights into the mechanisms of TB drug metabolism, drug action, and drugrelated side effects, thereby paving the way for the development of improved therapeutic approaches for the disease, and perhaps more importantly, also for monitoring treatment progression.
\end{abstract}

Keywords: pharmacometabolomics, tuberculosis, urinary metabolome, precision medicine, biomarkers, DOTS

\section{Introduction}

$\mathbf{T}$ UBERCULOSIS (TB), AN AIRBORNE DISEASE caused by Mycobacterium tuberculosis (M. tuberculosis) infection, is currently listed by the World Health Organization (WHO) as one of the top 10 causes of disease-related deaths worldwide (WHO, 2018). This disease is, furthermore, ranked above human immunodeficiency virus (HIV)/acquired immune deficiency syndrome as the leading cause of death from a single infectious agent. In 2017 alone, an estimated 10 million people were newly infected with $M$. tuberculosis, with $\sim 1.3$ million deaths among HIV-negative patients (WHO, 2018).

For the treatment of active TB, the WHO currently recommends the 6-month directly observed treatment, short-course (DOTS) treatment program. This treatment program consists of two phases, including an initial, intensive 2-month phase, which entails the coadministration of four first-line drugs, isoniazid (INH), rifampicin (RIF), pyrazinamide (PZA), and ethambutol (EMB), followed by the second phase, including only RIF and INH, for a further 4 months (WHO, 2018).

When DOTS is fully adhered to, the program has a high cure rate in patients with drug-susceptible TB, and in the years 2000 2018, this therapeutic approach has successfully cured and saved the lives of roughly 60 million individuals (WHO, 2018). The latest treatment outcome data gathered from the WHO, however, stated that up to $45 \%$ of multidrug-resistant and $18 \%$ of drug-susceptible TB cases were still treated unsuccessfully in 2015 and 2016, respectively (WHO, 2018). Several factors, including varying individual drug metabolism, HIV coinfection, and/or limited access to medical facilities where these drugs are administered, have been associated with a failed treatment outcome and the development of drug resistance.

Another major contributor to TB treatment failure is the disruption or premature termination of this first-line treatment approach, mainly ascribed to drug-related side effects (De Villiers and Loots, 2013). These side effects (collectively

\footnotetext{
${ }^{1}$ Human Metabolomics, North-West University, Potchefstroom, South Africa.

${ }^{2}$ Division of Molecular Biology and Human Genetics, Faculty of Medicine and Health Sciences, DST/NRF Centre of Excellence for Biomedical Tuberculosis Research/MRC Centre for Molecular and Cellular Biology, Stellenbosch University, Tygerberg, South Africa.

${ }^{3}$ Mater Research Institute, The University of Queensland, Brisbane, Australia.
} 
occurring in $\sim 21 \%$ of patients enrolled in DOTS therapy) range from minor skin and body fluid discoloration and headaches, to more severe side effects including hepatotoxicity, neurotoxicity, and gastrointestinal complications (Nanda et al., 2016).

Furthermore, TB recurrence after successful treatment, may also manifest either through reinfection with a different $M$. tuberculosis strain to that originally cured, or a relapse infection from the original strain (Lambert et al., 2003). The incidence of recurrence is highly variable and roughly ranges from 5\% to 47\% (Mirsaeidi and Sadikot, 2018), and various factors, including, HIV infection and diabetes, inadequate treatment protocols, disease severity, age, a male gender, silicosis and pre-existing resistance to the drugs administered, have been associated with an elevated recurrence prevalence (Lambert et al., 2003; Mirsaeidi and Sadikot, 2018).

From the above, it is evident that, as part of the fight against TB, the occurrence of TB treatment failure and relapse needs to be urgently addressed. The first step toward achieving this goal would be to gain a better understanding of the TB drug mechanisms, and to alternatively elucidate novel mechanisms related to drug metabolism, drug action, and drug toxicity, through the application of newer research fields, such as pharmacometabolomics.

Metabolomics is a relatively new "omics" domain and can be defined as the unbiased approach of studying the metabolome profiles within a biological system (Everett et al., 2013). When metabolomics is implemented toward identifying metabolome variations and adaptations induced by a genetic perturbation (such as a mutation) or an environmental factor (such as nutritional status or a disease state), the research approach is termed metabolomics (Nicholson et al., 1999). In the case where the environmental perturbation being studied is a specific drug or drug combination, the research domain is reclassified as pharmacometabolomics. In such studies, the identified metabolome variations will typically include the addition of drugderived metabolites, originating directly from the xenobiotic metabolism, to the metabolome profiles, and may also represent metabolite alterations to the normal human metabolome in response to treatment (Koen et al., 2016).

Many research groups have successfully applied pharmacometabolomics methodologies to investigate various aspects of the first-line TB drugs included in the DOTS treatment regime, using a variety of research models and analytical apparatus (Du Preez and Loots, 2018; du Preez et al., 2019). Although most of these studies focused on elucidating new, or elaborating on known mechanisms of drug metabolism, drug action, drug toxicity, and drug resistance of individual TB drugs, very few research groups studied the effect of the combined drug therapy on the host metabolome.

When investigating the effects of the combination TB drug, Rifater, on the urinary metabolome of rats, metabolic abnormalities similar to that of patients with a multiple acyl-CoA dehydrogenase deficiency, was found. This observation suggests that Rifater results in an inhibition of the electron transport chain and subsequently explains many of the side effects associated with these first-line TB drugs (Loots et al., 2005).

In a related study, a gas chromatography mass spectrometrybased pharmacometabolomics approach could differentiate between the global urine metabolome profiles of healthy controls and TB patients, and a number of characteristic disease biomarkers were identified. In addition, the metabo- lome profiles of urine samples collected at different time intervals after the initial DOTS treatment grouped together in a time-dependent manner, with metabolome profiles shifting more toward that of healthy controls as treatment progressed (Das et al., 2015). This observed trend was further investigated in a follow-up study by specifically exploring the known urinary metabolites of INH, RIF, PZA, and EMB, and how the concentrations of these drug metabolites changed over time. A novel drug metabolite of EMB was identified with this approach (Das et al., 2016).

Recently, pharmacometabolomics was able to identify new toxic mechanisms associated with the ingestion of firstline TB drugs by comparing the altered urinary metabolomes of TB patients on DOTS therapy with that of newly diagnosed TB patients who were not on treatment as yet, and additionally, predictive markers for developing hepatotoxicity were identified (Cao et al., 2018).

Building on this, we aimed to use a two-dimensional gas chromatography time-of-flight mass spectrometry (GCxGCTOFMS)-based pharmacometabolomics approach, to identify those metabolites better describing the alterations in the human urinary metabolome induced by TB therapy and the degree of these alterations at various time points throughout treatment. The obtained results could improve the understanding of firstline TB drug mechanisms of action, mechanisms of toxicity, and also the adaptations of the host to TB drug toxicity.

\section{Materials and Methods}

\section{Patient recruitment and sample collection}

Ethical approval was granted for the collection and use of these samples by the respective ethics committees of the University of Stellenbosch (no. 99/039) and the North-West University (no. NWU-00127-11-A1). Informed consent from each participant for the use of their urine for research purposes was also obtained, and all samples were anonymized immediately to maintain confidentiality.

Patient urine samples were collected in standard vials by the NRF/DST Centre of Excellence for Biomedical Research, University of Stellenbosch, from culture-confirmed TB patients recruited from rural communities in the Western Cape, South Africa. The sampling of patient urine took place at the time of diagnosis (before treatment onset) and again at various intervals during the intensive phase of DOTS treatment (weeks 1,2, and 4). These sample collection time points were deemed significant in a preceding study, where distinct cytokine biosignatures, characterizing early treatment response, were identified (Ronacher et al., 2019). Samples were received from all patients at all time intervals.

After collection, samples were immediately frozen and transported on dry ice to the North-West University (Human Metabolomics: Infectious Disease Laboratory), for metabolomics analysis, where they were stored at $-80^{\circ} \mathrm{C}$ before extraction. A total of 23 drug-susceptible TB patients (11 men and 12 women) who were not pregnant nor diagnosed with other diseases (such as diabetes, cancer, chronic bronchitis, or sarcoidosis) were included in this study. All patients were HIV seronegative and did not receive any additional medication. The patients had a mean age of $35 \pm 10.68$ years and body mass index of $18 \pm 2.11 \mathrm{~kg} / \mathrm{m}^{2}$. Only patients with a culture-confirmed cured treatment outcome after the 6-month treatment were used in this study. 


\section{Quality control samples}

To ensure precision and accuracy of the data obtained (i.e., instrument and extraction method repeatability), an in-house quality control (QC) sample, representing a combination of samples previously collected from healthy individuals, was used. One aliquot of this master QC sample was extracted and derivatized with each batch of samples analyzed and injected onto the GCxGC-TOFMS at the beginning, middle and end of each batch.

\section{Reagents and chemicals}

Hexane, 3-phenylbutyric acid, and $O$-bis(trimethylsilyl)trifluoroacetamide (BSTFA) were purchased from SigmaAldrich (St. Louis, MO, USA). Hydrochloric acid ( $\mathrm{HCl})$, pyridine, sodium sulfate anhydrous $\left(\mathrm{Na}_{2} \mathrm{SO}_{4}\right)$, and trimethylchlorosilane (TMCS) were purchased from Merck (Darmstadt, Germany). The ultrapure Burdick and Jackson brand organic solvents, ethyl acetate, and diethyl ether were obtained from Honeywell International, Inc. (Muskegon, MI, USA).

\section{Organic acid extraction}

The creatinine values for all urine samples, including that of the QC samples, were determined with a creatinine enzyme kit (Thermo Scientific; reference number 981845) and analyzed on an Indiko Clinical Analyser, Type 863 (Thermo Scientific). Organic acid extraction of the urine was performed as previously described, with slight modifications (Luies and Loots, 2016). In short, the specified amount of each urine sample (determined in accordance to the creatinine values) was aliquoted into a salinized glass tube (Kimax) and acidified by the addition of six drops of concentrated $\mathrm{HCl}$. As an internal standard, 3-phenylbutyric acid $(25 \mu \mathrm{mol} /$ mg creatinine) was added to each sample.

This compound was chosen as the internal standard because it is absent in human urine, elutes almost in the middle of an organic acid profile, and is known to coelute with very few other organic acids (Reinecke et al., 2012).

Hereafter, $6 \mathrm{~mL}$ ethyl acetate was added to each sample, followed by mixing and centrifugation $(1050 \times g$ for $3 \mathrm{~min})$ to achieve solvent phase separation. The resultant organic phase was then aspirated with a Pasteur pipette and transferred to a clean Kimax test tube. The remaining aqueous phase was once again extracted with $3 \mathrm{~mL}$ diethyl ether and after centrifugation, the organic solvent was added to that previously collected. $\mathrm{Na}_{2} \mathrm{SO}_{4}(\sim 3 \mathrm{~g})$ was then added to the collected organic solvent to remove any residual water that might have been present, followed by a brief vortex and centrifugation step. The newly obtained organic phase was subsequently decanted from the pellet and evaporated to complete dryness under a stream of nitrogen at $37^{\circ} \mathrm{C}$.

\section{Derivatization}

For derivatization purposes, BSTFA $(22.6 \mu \mathrm{L} / \mu \mathrm{mol}$ creatinine), TMCS ( $4.5 \mu \mathrm{L} / \mu$ mol creatinine $)$, and pyridine $(4.5 \mu \mathrm{L} /$ $\mu$ mol creatinine) were added to the dried extracts for a derivatization period of $60 \mathrm{~min}$ at $60^{\circ} \mathrm{C}$ (Luies and Loots, 2016). Hereafter, each derivatized sample was transferred to a GC-MS vial containing a vial insert.

\section{GCXGC-TOFMS analysis}

After derivatization, samples were placed randomly into the Gerstel MultiPurpose Sampler tray (Gerstel GmbH and Co. KG, Mülheim an der Ruhr, Germany) for chromatographic analyses. This autosampler is equipped with a Pegasus 4D GCxGC-TOFMS (LECO Africa (Pty) Ltd., Johannesburg, South Africa). All samples ( $1 \mu \mathrm{L})$, including QC samples, were injected onto the GC at a split ratio of 1:12. Helium was used as the carrier gas at a constant flow rate of $1 \mathrm{~mL} / \mathrm{min}$ and the injector temperature was maintained at $280^{\circ} \mathrm{C}$ for the entire run.

Compound separation was achieved using a Restek Rxi5Sil MS primary capillary column $(30 \mathrm{~m}, 0.25 \mu \mathrm{m}$ film thickness, and $250 \mu \mathrm{m}$ internal diameter), programmed at an initial temperature of $50^{\circ} \mathrm{C}$ for $1 \mathrm{~min}$, followed by an increase of $5^{\circ} \mathrm{C} / \mathrm{min}$ to a temperature of $120^{\circ} \mathrm{C}$, then an increase of $7^{\circ} \mathrm{C} / \mathrm{min}$ to a temperature of $280^{\circ} \mathrm{C}$, at which it was maintained for $4 \mathrm{~min}$ and then finally increased at a rate of $20^{\circ} \mathrm{C} /$ min to a final temperature of $300^{\circ} \mathrm{C}$, at which it was maintained for a further $1 \mathrm{~min}$. For secondary separation, a Rxi-17 capillary column $(0.9 \mathrm{~m}, 0.1 \mu \mathrm{m}$ film thickness and $100 \mu \mathrm{m}$ internal diameter) was used, and the oven was programmed to have an identical temperature gradient to that of the primary column, only with an offset of $+5^{\circ} \mathrm{C}$ at all the time points.

Cryomodulation was achieved with a hot pulse of nitrogen gas for $0.7 \mathrm{sec}$, every $3 \mathrm{sec}$, to ensure control of the effluent emerging from the primary column onto the secondary column. The filament was switched off for the first $8 \mathrm{~min}$ of each sample run as this period was considered to correspond to a solvent delay, during which no mass spectra were recorded. This 8-min interval was, however, included in the first column's time axis to reflect accurate compound retention times. The transfer line temperature was held at a constant $270^{\circ} \mathrm{C}$ and the ion source temperature at a constant $230^{\circ} \mathrm{C}$ for the entire run. The detector voltage was $1600 \mathrm{~V}$ and the filament bias $-70 \mathrm{eV}$. Mass spectra were acquired at a rate of 100 mass spectra per second, over the range $50-600 \mathrm{~m} / \mathrm{z}$.

\section{Data processing}

Mass spectral deconvolution, peak alignment, and peak identification were performed using ChromaTOF software (version 4.32) from Leco Corporation. Deconvolution was performed at a signal-to-noise ratio of 50, with a minimum of two apexing peaks. To eliminate the effect of retention time shifts, Statistical Compare (an optional ChromaTOF function) was used to align peaks with similar mass spectra and retention times, and the identities of these peaks were determined by comparing them to commercially available [NIST (National Institute of Standards and Technology)] and in-house-generated mass spectral libraries. A data matrix consisting of all the compounds identified in all the analyzed samples was subsequently generated and peak areas were normalized relative to the internal standard to eliminate potential variation because of irregularities in the extraction process.

\section{Data cleanup}

Before statistical data analysis, data cleanup was carried out to remove potential contaminants and background noise. Urine samples from individual patients (collected at weeks 0 , 1, 2 and 4) were paired, and compounds that were not present 
in $80 \%$ of the samples collectively, were removed using an $80 \%$ zero filter. Since the majority of the zero values present in the data can be ascribed to an undetectable, low abundance, rather than truly being absent, the remaining zero values were replaced by random numbers drawn from a uniform distribution between one-third and two-thirds of the minimum nonzero value.

After this, log transformation (with a shift parameter of one) and Pareto scaling were performed separately for each variable. Log transformation was applied to correct for the positively skewed distribution associated with metabolomics data. Pareto scaling is a scaling method similar to that of auto scaling, but instead of the standard deviation, the square root of the standard deviation is used as a denominator. Pareto scaling can be sensitive to large fold changes, but it stays closer to the original measurement than the auto scaling method (Van den Berg et al., 2006). After the application of these cleanup steps, a total of 770 metabolites remained in the dataset that was used in the subsequent statistical analyses.

\section{Statistical analysis}

Quality assurance. Aliquots from the pooled QC sample were used to establish whether the samples were consistently analyzed within and across batches. To ensure the absence of possible batch effects in the generated data, a principle component analysis (PCA) scores plot, summarizing the projection of the variation within the total dataset, was generated using MATLAB with Statistics Toolbox (2012b) (The MathWorks, Inc., MA, USA) and PLS Toolbox 8.2.1 (2016) (Eigenvector Research Software available at www.eigenvector .com). The generated scores of the analyzed samples (QCs and all analyzed patient samples, consisting of those collected at weeks $0,1,2$, and 4) were color coded to visually compare the variation between the QC samples to that of the patient samples.

Metabolite marker identification. To select the most significant metabolite markers, two analyses of variance (ANOVA) tests, including the repeated-measures (RM) ANOVA and ANOVA simultaneous component analysis (ASCA) were performed on the "cleaned" dataset using MATLAB with Statistics Toolbox (2012b) and PLS Toolbox 8.2.1 (2016) (The MathWorks, Inc.).

RM-ANOVA calculated and subtracted the factor level for the time points, and for each case, aiming to identify the metabolites showing significant $(p \leq 0.0001)$, treatmentrelated variation across all time points (Tabachnick and Fidell, 2007). To control the family-wise error rates during multiple hypotheses testing, the Bonferroni-Holm correction was applied. All metabolites with a Bonferroni-Holm adjusted $p \leq 0.1$ were deemed significant.

ASCA, a tool typically used for the analyses of complex metabolomics datasets, identifies dominant patterns of variation associated with each factor and investigates the interactions of these factors (Smilde et al., 2005). The loadings of the ASCA can be used to identify a list of metabolites with a discriminatory ability either independently or in combination with other metabolites. Metabolites with a sum of the squared loadings (SSLs) above the threshold (0.0075) for the first two levels of variance (LV) were considered significant. This threshold represents the point where a significant reduction in SSL could be observed after visual inspection of a scree plot of the SSL.
To compile a list of metabolite markers, which are considered to be those compounds that best describe the variation between the pretreatment and during treatment patient groups, the significant metabolites identified by RMANOVA and ASCA were combined.

\section{Results}

\section{Quality assurance}

Quality assurance was carried out using PCA, to determine any variation between the QC samples and that of the patient samples analyzed. Figure 1 represents the scores plot of the first three PCs, with PC1 explaining 23\%, PC2 explaining $11 \%$, and PC 3 explaining $6 \%$ of the total variation in the data. From these results, it is clear that a negligible batch effect in the total dataset exists when comparing the QC with the patient sample data, and therefore, no batch effect correction was performed.

\section{Urinary metabolome variations induced by DOTS therapy}

The scores plot emanating from the ASCA (Fig. 2) shows a clear differentiation between the patient samples collected before treatment onset, and those collected during treatment. From this result, it is evident that DOTS treatment induces significant variations to the urinary metabolome profiles of TB patients, from as early as 1 week after the initial dosage. Furthermore, samples collected at various intervals during treatment also grouped according to the specific collection time point, thereby indicating a time trend in the drug-induced metabolome variations. The amount of variance explained by LV1 was $44 \%$ and LV2 was $33 \%$.

A total of 93 metabolites (identified with RM-ANOVA only [ $n=44]$, with ASCA only [ $n=16]$, and with both RMANOVA and ASCA [ $n=33]$ ) were determined to be significant markers, characterizing the effect of treatment on the urinary metabolomes of TB patients. The identities of 39 of these markers (Table 1) could be validated by comparing their mass spectrums with those available in commercial and in-house mass spectral libraries, generated from previously injected compound standards. Further investigation of these markers indicates that these can be grouped as: (1) metabolites originating directly from the ingested TB drugs, (2) metabolites indicating a decreased $M$. tuberculosis load, and (3) an altered human metabolome because of the host's response to treatment (Table 1).

\section{Discussion}

\section{Metabolites originating directly from the ingested TB drugs}

PZA, and one of its major metabolites, pyrazinoic acid (POA), formed through the de-amination of the parent drug (Ramappa and Aithal, 2013) and excreted through the kidneys (Saukkonen et al., 2006), were identified as significant markers and remained elevated throughout the first 4 weeks of therapy (Table 1), confirming observations made by Das et al. (2016). PZA is a sterilizing drug that inhibits actively growing $M$. tuberculosis after being converted to its active 


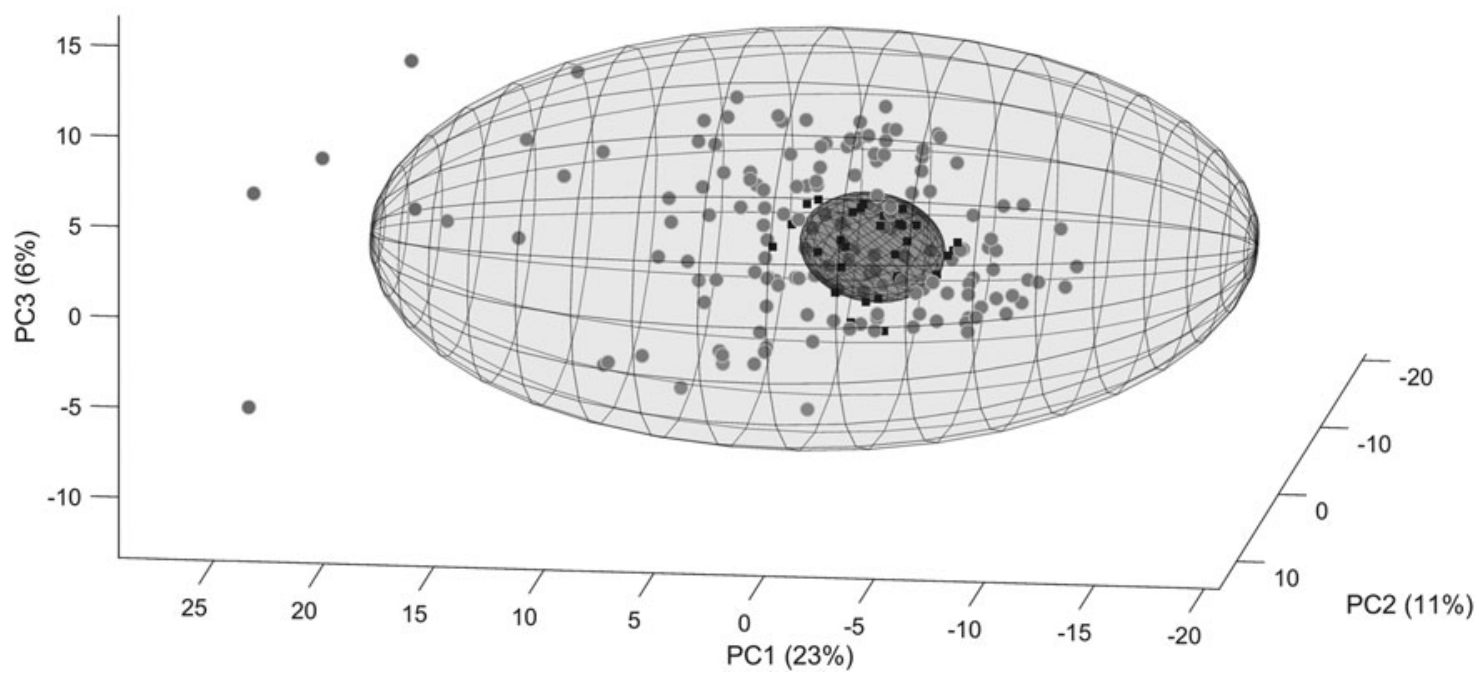

FIG. 1. Three-dimensional PCA scores plot showing the variation between the QC samples in relation to the variation between the patient samples. PCA, principal component analysis; QC, quality control.

form, POA, through the bacterial enzyme, pyrazinamidase. Therefore, although the elevated urinary POA levels detected in this study can mainly be ascribed to the human xenobiotic metabolism of PZA, fractions of this metabolite might also originate as a result of bacterial drug activation.
A similar concentration trend to that detected for PZA and POA over the course of therapy was observed for citric acid ethyl ester, dodecyl acrylate, and hippuric acid (Table 1), indicating a possible direct relation to the ingested TB drugs. Citric acid ethyl ester is used as an inactive ingredient in

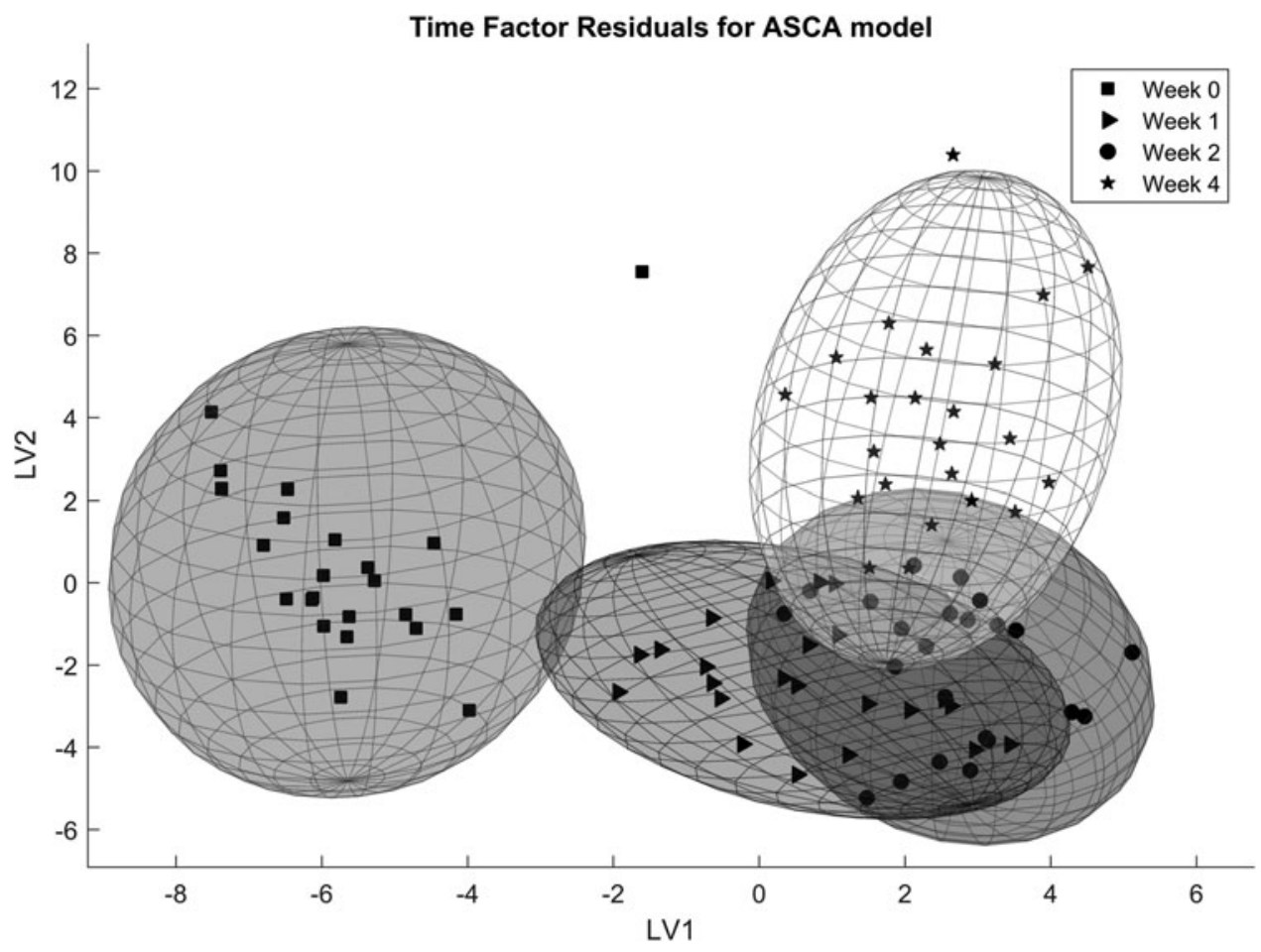

FIG. 2. ASCA scores plot of the sum of effects and projected residuals of the metabolomics data obtained from tuberculosis patients before treatment onset (week 0), and at various intervals during directly observed treatment, short-course treatment. ASCA, analyses of variance simultaneous component analysis; LV, level of variance. 


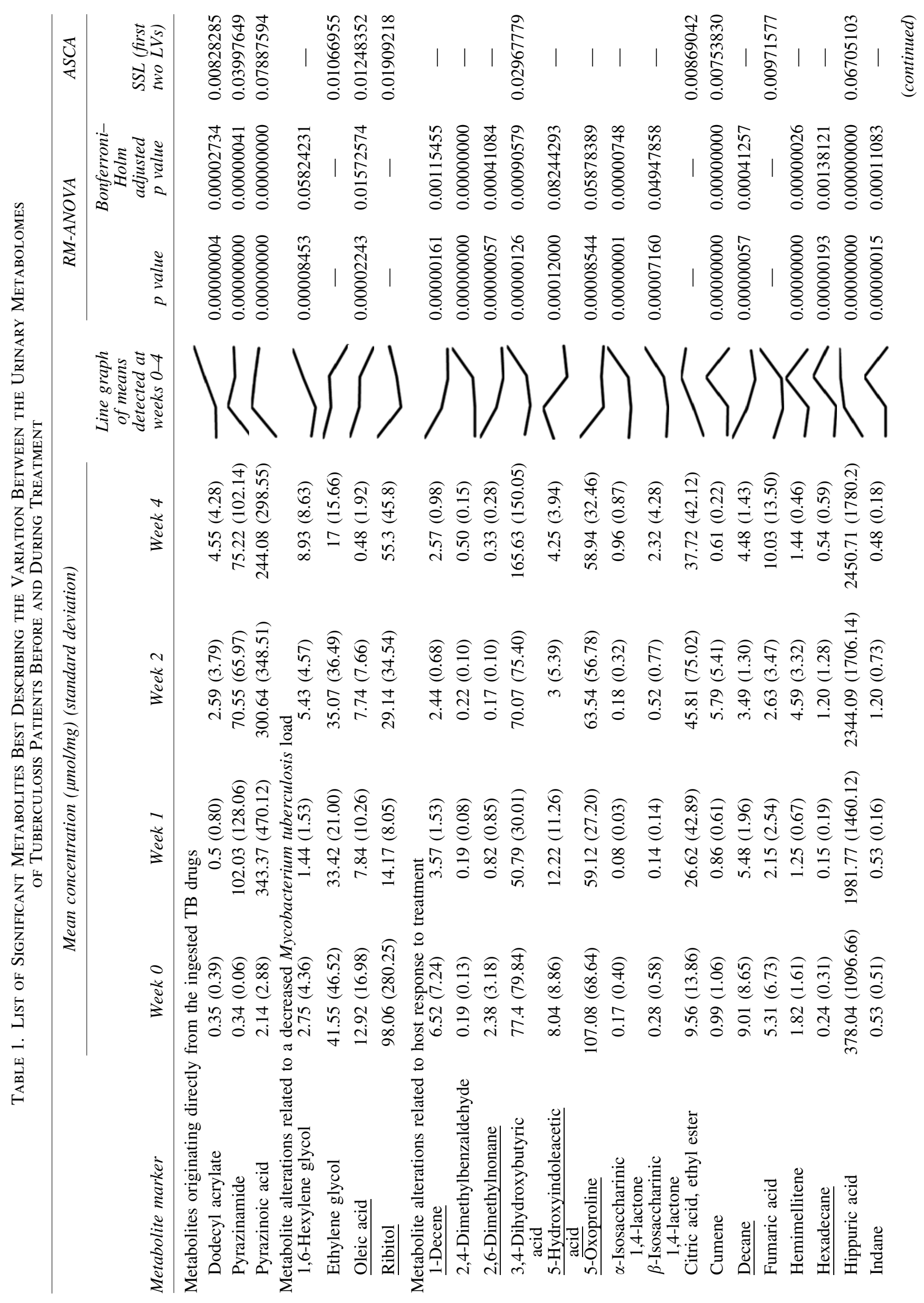




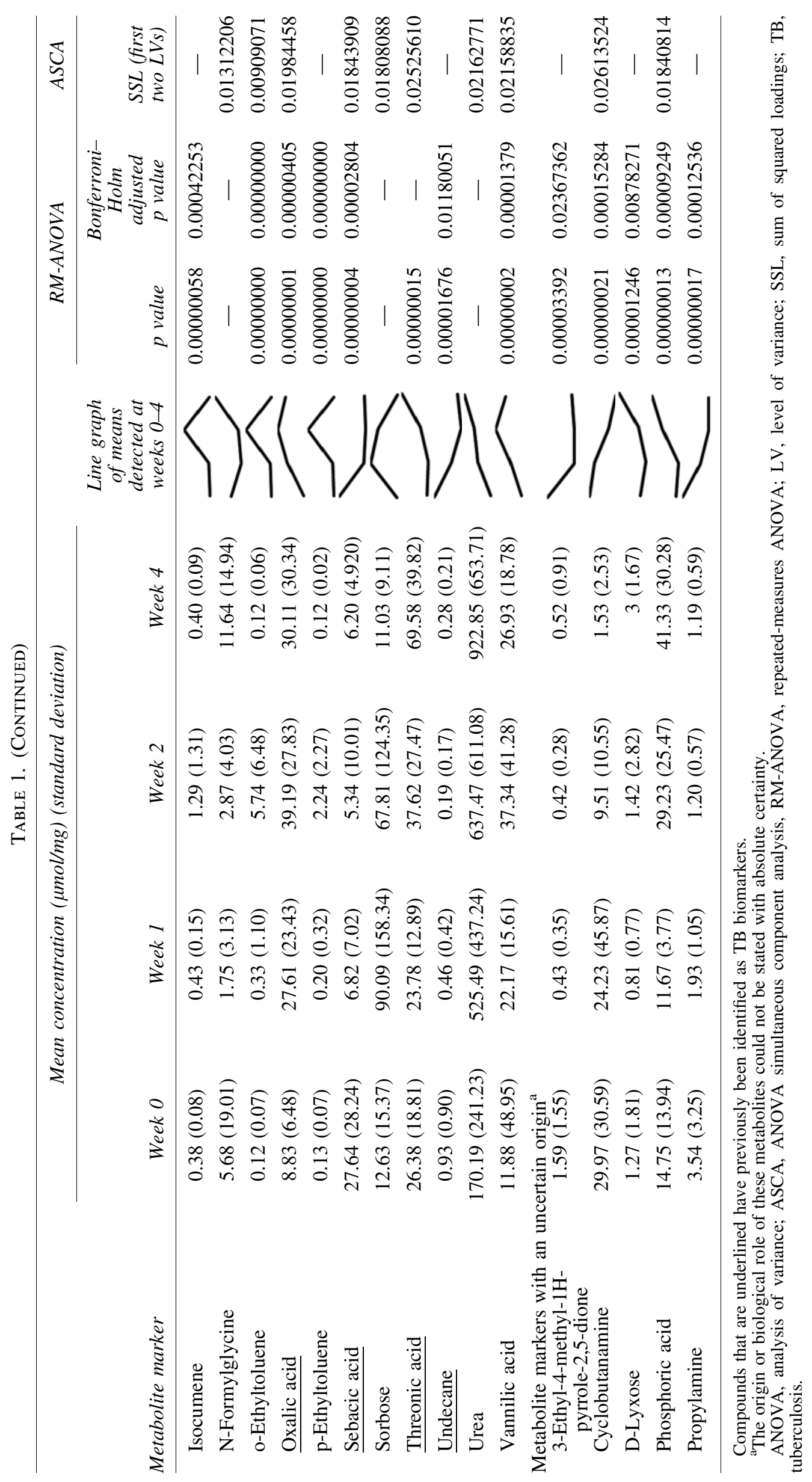


various medicine capsules (Pole et al., 2016), and likewise, dodecyl acrylate is copolymerized with acrylic acid as a drug coating allowing for drug release in specific $\mathrm{pH}$ environments (Ngwuluka, 2010). Similarly, the observed concentration trend for hippuric acid, a normal component of urine that typically elevates in concentration with increased consumption of phenolic compounds (Gatley and Sherratt, 1977), could be related to the use of sodium benzoate as a lubricant during pharmaceutical manufacturing of these tablets and capsules (Varma, 2016).

Therefore, although we could not confirm or test the content of the coatings of the drugs ingested by the study participants, it is highly likely that these compounds, which are well-known ingredients used during drug manufacturing, may originate from these capsules/coatings. That being said, the elevated concentrations of citric acid ethyl ester and hippuric acid levels detected in the during-treatment urine samples may additionally be a consequence of drug-induced enzyme inhibition, as discussed later.

\section{Metabolite alterations related to a decrease in the M. tuberculosis load}

During the course of a successful TB treatment regimen, metabolites originating directly from $M$. tuberculosis bacilli are expected to decrease over the duration of the treatment short course. Accordingly, ribitol, a metabolite directly associated with the cell walls of mycobacteria (Mikusova et al., 1996; Silhavy et al., 2010), and oleic acid, one of the most abundant fatty acids present in M. tuberculosis (Olivier and Loots, 2012), were observed to follow this trend (Table 1). Confirmatory to this observation, elevated concentrations of urinary ribitol (Luies and Loots, 2016) and oleic acid from sputum collected from TB patient, has previously been identified as biomarkers for TB disease (Du Preez and Loots, 2013), which further substantiates possible diagnostic or treatment progression monitoring applications using these markers.

Polyethylene glycol (PEG) chains are present in the acylbinding channels of KasA, a key enzyme involved in the biosynthesis of long-chain fatty acids and mycolic acids that forms part of the fatty acid synthase II system in M. tuberculosis (Luckner et al., 2009). The bound PEG molecule (a polymer of ethylene glycol) mimics an acyl chain of $\sim 40$ carbon atoms in length during fatty acid elongation in $M$. tuberculosis. In humans, PEG, which is considered a toxin, is metabolized through alcohol dehydrogenase (ADH) and various other liver enzymes, to produce formic acid, oxalic acid, and hippuric acid (Walder and Tyler, 1994).

In the case of PEG poisoning, ethanol, a competitive inhibitor of $\mathrm{ADH}$, is considered a treatment option. In this case, ethanol, which has a higher affinity for ADH than PEG, will saturate the enzyme, thereby inhibiting PEG metabolism, subsequently resulting in the excretion of intact PEG (and not ethylene glycol) in patient urine (Herold et al., 1989). Although considered a weaker substrate than ethanol, EMB is also metabolized through ADH to form EMB-aldehyde (Peets and Buyske, 1964; Strolin Benedetti et al., 2006) (Fig. 3). Because a significant decrease in ethylene glycol and a significant increase in hexylene glycol were detected in the urine of TB patients during the course of the treatment regimen (Table 1), we propose that the high abundance of EMB in these patients imitates the effect that ethanol has on $\mathrm{ADH}$ during PEG poisoning, thereby limiting the decomposition of the PEG originating from M. tuberculosis (Fig. 3).

Further evidence supporting this is that EMB is an excellent chelating agent, which is able to form stable complexes with essential trace metals such as copper, zinc, and iron, and hence it is proposed that EMB administration could affect the normal functioning of various metal requiring enzymes, such as ADH, for example (Sarkar et al., 2016). The effect of EMB on ADH will consequently also influence the metabolism of other alcohols, including ethanol, which is discussed in more detail in the following section.

\section{Metabolite alterations reflecting changes to the human metabolism in response to therapy}

Oxidative stress usually occurs because of an imbalance between reactive oxygen species (ROS) production and the counter effect of the antioxidant mechanisms. Although oxidative stress results in damage to the host's cells, it is also a means by which the host eliminates a threat of an infectious agent, by inducing DNA, protein, and lipid damage (Shastri et al., 2018; Van Acker and Coenye, 2017). During M. tuberculosis infection, the host would respond by initiating a respiratory burst within the infected macrophages, resulting in high levels of ROS. Mycobacteria do, however, also have antioxidant mechanisms, and therefore, to kill the bacteria, the ROS produced by the host needs to be enough to overwhelm the bacterial antioxidant defense systems (Jamaati et al., 2017).

Elevated oxidative stress levels are, therefore, a wellknown occurrence in TB patients (Jamaati et al., 2017; Lamichhane, 2011; Nicholson et al., 1996), subsequently explaining the elevated levels of lipid peroxidation products, including sebacic acid and several hydrocarbons (decane, 1decene, 2,6-dimethylnonane, hexadecane, and undecane), which were previously identified as TB markers in patient sputum (Du Preez and Loots, 2013), in the pretreatment patient urine samples (Table 1).

Similarly, 5-oxoproline, a product of glutathione metabolism (Fig. 3) (Bando et al., 2011), also previously identified as a biomarker for active TB and pathological lung damage (Che et al., 2013), was detected in comparatively elevated levels in the urine samples collected before treatment onset (Table 1). This indicates an upregulated synthesis of glutathione, the most prevalent natural antioxidant used to systemically detoxify ROS, in the untreated TB patients (Biswas and Rahman, 2009). The elevated levels of oxalic acid detected in the urine samples collected after treatment commenced, is ascribed to glyoxylic acid synthesis, which further supports the notion of an activation of the systemic antioxidant response of the host (Holmes and Assimos, 1998).

Ascorbic acid (vitamin C) is a known natural antioxidant and an essential vitamin obtained through dietary intake (Smirnoff, 2018). Apart from its antioxidant function, ascorbic acid can also act as a prooxidant during M. tuberculosis infection by driving the Fenton reaction toward the formation of highly reactive hydroxyl radicals (Fig. 3) (Vilcheze et al., 2013). This oxidative burst generation makes $M$. tuberculosis highly susceptible to killing by ascorbic acid (Shastri et al., 2018; Vilcheze et al., 2013) and the reduced levels of threonic acid and oxalic acid, both normal oxidation products of ascorbic acid, detected in the pretreatment urine samples, could, therefore, be a reflection of the use of this 


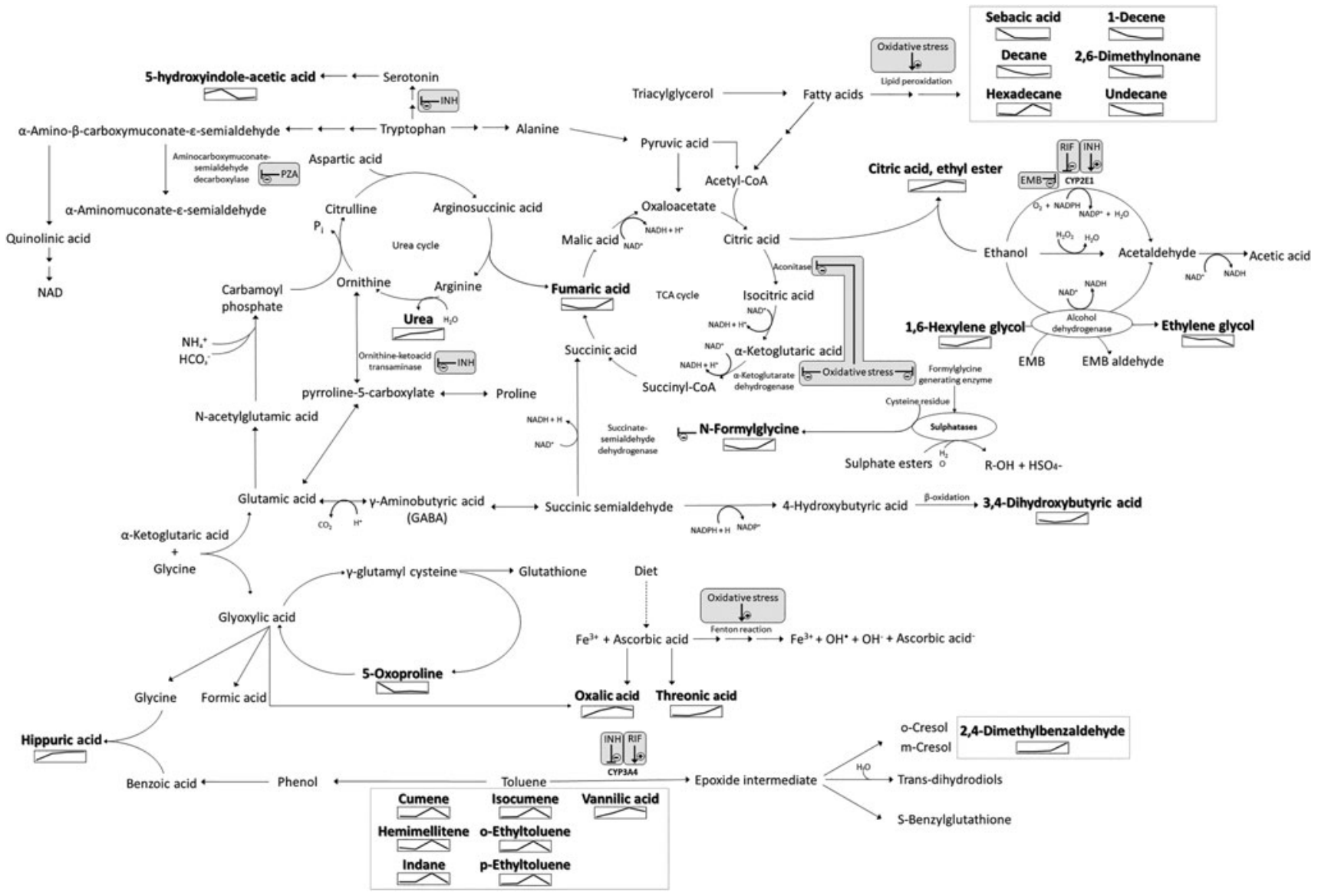

FIG. 3. Schematic representation of the altered urinary organic acid metabolome induced by therapy. Metabolites identified as significant markers in this study are indicated in bold, accompanied by a line graph of the mean concentration of this compound at weeks $0-4$ of treatment. Inhibition is indicated with a minus sign, Induction with a plus sign. CYP, cytochrome P450; EMB, ethambutol; INH, isoniazid; NAD/NADH, nicotinamide adenine dinucleotide; PZA, pyrazinamide; RIF, rifampicin; TCA, tricarboxylic acid.

vitamin in the host's fight against the infection by the Fenton reactions.

A steady reduction in the abundance of the aforementioned lipid peroxidation products and 5-oxoproline, and in addition elevated levels of threonic acid and oxalic acid, was subsequently seen as treatment progressed over the 4week period. These observations can be ascribed to a significant drop in the host's oxidative stress levels during treatment, which could directly be ascribed to a decreased mycobacterial load and consequent fading of the disease phenotype, in response to therapy.

The formylglycine-generating enzyme (FGE) is an oxidoreductase, and functions by post-translationally activating sulfatase enzymes, thereby producing formylglycine as a by-product (Fig. 3) (Knop et al., 2017). FGE uniquely uses molecular oxygen, without the need of an oxygen activating cofactor such as FAD (flavin adenine dinucleotide). This enzyme is also sensitive to inactivation by ROS in the endoplasmic reticulum (Dierks et al., 2005). Furthermore, Knop et al. (2017) recently indicated an association between oxidative stress and FGE activity in humans.

In our investigation, formylglycine was detected in comparatively reduced concentrations before treatment onset, indicating a degree of FGE inactivation, as a result of increased oxidative stress induced by $\mathrm{TB}$, with a sharp increase between weeks 2 and 4 of treatment (Table 1), indicating a reactivation of this enzyme, and furthermore confirming overall reduced systemic oxidative stress in the host during the course of successful TB treatment.

Elevated concentrations in 3,4-dihydroxybutyric acid (Table 1), similar to that seen in patients with a succinic semialdehyde dehydrogenase (SSADH) deficiency (Shinka et al., 2002), was observed in the urine of the treated TB patients. The inhibition of SSADH is known to result in the accumulation of succinic acid semi-aldehyde, and its metabolite, 4-hydroxybutyric acid, which in turn forms 3,4-dihydroxybutyric acid through fatty acid $\beta$-oxidation (Fig. 3). In previously published literature, formylglycine has been identified as an inhibitor of plant and fungal SSADH (Kumar and Punekar, 1998), and our results suggest a similar inhibition in humans, because identical treatment-induced concentration trends were detected for formylglycine and 3,4-dihydroxybutryric acid.

SSADH activity is therefore indirectly regulated by oxidative stress levels through FGE, and during a state of the elevated levels of oxidative stress would therefore promote the fuelling of the tricarboxylic acid (TCA) cycle by succinic acid semialdehyde (Fig. 3).

The previously described elevated levels of oxidative stress in TB (Jamaati et al., 2017; Lamichhane, 2011; Nicholson et al., 1996) are also well known to inhibit 
various TCA cycle enzymes, of which aconitase, followed by $\alpha$-ketoglutarate dehydrogenase, are the two mostly affected (Tretter and Adam-Vizi, 2000). This inhibition could partially be responsible for the low abundance of fumaric acid and citric acid (detected as citric acid ethyl ester, formed by citric acid's esterification from ethanol) observed in the pretreatment urine samples, followed by the gradually elevating concentrations of these compounds as therapy commences, and the oxidative stress levels dissipates (Table 1).

Furthermore, ethanol catabolism in humans can be achieved by numerous pathways, including degradation by ADH during normal intermitted consumption, and degradation by cytochrome P450 (CYP) 2E1, the latter being activated during states of chronic alcohol ingestion (Lieber, 1997).

As mentioned earlier, EMB affects the normal functioning of ADH by acting as a chelating agent, and the administration of EMB during therapy would subsequently be expected to prevent ethanol metabolism through $\mathrm{ADH}$, resulting in elevated concentrations of systemic ethanol. The activity of CYP2E1, in turn, is also influenced by TB drugs, with INH being a potent inducer, and RIF and EMB being strong inhibitors of this enzyme (Lee et al., 2014; Pelkonen et al., 2008; Takeda et al., 2000). It has also been shown that CYP2E1 enzyme induction through RIF and EMB is not an immediate effect, and will only reach an optimal response $\sim 3$ weeks after the initial administration of the inducing agent, whereas inhibition of the enzyme complex is far quicker, and typically takes place 2-3 days after the administration of an inhibitory agent (Swart and Harris, 2005).

Considering this, the initial increase in the concentrations of citric acid ethyl ester immediately after treatment has commenced is partially related to the predominant inhibitory effects exerted by EMB and RIF, preventing ethanol degradation, and promoting ethanol esterification with citric acid (Fig. 3). After 2 weeks of treatment, the reduced citric acid ethyl ester concentration can be associated with INH enzyme induction of CYP2E1 and subsequently elevated ethanol degradation.

Apart from CYP2E1, CYP3A4 activity is also known to be affected by ingestion of first-line TB drugs, with INH being a weak inhibitor and RIF a potent inducer of this enzyme through the pregnane $X$ receptor (Swart and Harris, 2005). Similar to that of CYP2E1, an initial INH-related inhibition occurs, followed by a delayed induction of CYP3A4 by RIF, resulting in the concentration trend observed for various benzene compounds (cumene, hemimellitene, $o$-ethyltoluene, $p$-ethyltoluene, isocumene, indane, and vannilic acid). These compounds, showing an initial increase in concentration over the first 2 weeks of treatment, followed by a severe decrease in concentration hereafter (Table 1), are well known to be metabolized by CYP3A4, as seen in Figure 3, using toluene as an example.

Supporting this observation, the concentrations of 2,4dimethylbenzaldehyde, a product of pseudocumene metabolism through CYP3A4, was elevated 4 weeks after therapy has commenced, also suggesting a CYP3A4 induction between weeks 2 and 4 of treatment. The increase in the concentrations of the phenolic compounds described previously, also contributes to the accumulation of hippuric acid detected in the during-treatment patient urine (Fig. 3), as discussed earlier.

The urea cycle is reportedly upregulated by hydrazine, a toxic INH metabolite (Bando et al., 2011; Nicholls et al., 2001), and accordingly, an increase in urea and fumaric acid, which are intermediates of this cycle (Watford, 2003) (Fig. 3) were observed in the samples collected after treatment has commenced (Table 1). The upregulated urea cycle functions to remove excess ammonia, which in this case, can be ascribed to increased catabolism of amino acids used as metabolic fuel in pathways such as the glutathione and lysine metabolisms (Bando et al., 2011).

The ingestion of PZA has been associated with an elevated conversion ratio of tryptophan to niacin, because of an inhibition of aminocarboxymuconate-semialdehyde decarboxylase (Shibata et al., 2001). This inhibitory effect would lead to a reduction in the serotonin substrate, tryptophan, and subsequently the reduced 5-hydroxyindole acetic acid (the breakdown product of serotonin by 5-hydroxyindole acetaldehyde), as was also detected in the urine of the patients receiving treatment in this investigation (Fig. 3). Although not deemed significant, a similar concentration trend to that observed for 5hydroxyindole acetic acid over the 4 weeks of treatment, was also observed for tryptophan, further substantiating this.

In addition, because INH is considered to be serotonergic (Demartini et al., 2013), it too would contribute to the reduced levels of 5-hydroxyindoleacetic acid detected in the treated patients. In addition, 5-hydroxyindole acetic acid was recently detected in significantly higher concentrations in urine samples collected from TB patients, than in their healthy control counterparts (Luies and Loots, 2016). This occurrence was ascribed to the impaired insulin production, and the consequent elevated glucose levels in these patients. Subsequently, the reduction of 5-hydroxyindole acetic acid after commencing treatment in this investigation (Table 1) concurs with these previous findings.

Both the alpha and beta forms of isosaccharinic-1,4lactone were detected in elevated concentrations in the urine of the treatment group (Table 1). Similar to the aforementioned PZA metabolites, these compounds are excreted through the kidneys and are normally present in relatively low concentrations in the urine (Niwa et al., 1980). Isosaccharinic-1,4-lactone originates from cellulose degradation under alkali conditions (Charles et al., 2015), similar to that observed when cellulolytic bacteria, which forms part of the human gut microbiome, initiate plant cell-wall degradation (Flint et al., 2012). Previous studies have indicated that both the TB infection itself and the treatment thereof, drastically alters the hemostatic state and composition of the human gut microbiome (Hu et al., 2019; Namasivayam et al., 2018), leading to a perturbed gut microbe composition, which persists even after treatment has been successfully completed.

In addition, urinary sorbose concentrations (formed by microbial sugar fermentation within the gut [Sato et al., 2017; Würsch et al., 1979]) was detected in significantly higher concentrations during the course of TB treatment (Table 1), serving as further support of an altered microbial fermentation and a disturbed gut microbiome induced by TB drugs.

\section{Conclusions}

This study shows how pharmacometabolomics can be successfully used to identify TB drug-induced variations in the urinary metabolome of treated patients, at specific treatment intervals. Treatment-induced metabolome variations originating directly from the ingested drugs reduced mycobacterial load, and host response to treatment, were identified. 
Although the administration of INH, PZA, and RIF, alone or in combination, have been proven to cause elevated oxidative stress and lipid peroxidation in the host (Sodhi et al., 1997), our results suggest that this effect is far less compared with the oxidative stress brought about by the TB disease itself.

Metabolite markers associated with oxidative stress dramatically declined between weeks 2 and 4 of treatment, indicating a return to a healthier phenotype. This observation also leads to the speculation that increased systemic oxidative stress levels play a major role in the manifestation of the symptoms associated with $\mathrm{TB}$, which are alleviated by treatment and the consequent lowering of the $M$. tuberculosis load. The concentration trend of the identified metabolite markers over the first 4 weeks of treatment, additionally indicated an initial drug-induced inhibition of the major drug metabolizing enzymes, CYP3A4 and CYP2E1, through INH and both RIF and EMB, respectively, followed by a delayed induction of the same enzymes by RIF and INH correspondingly. A drug-induced time-dependant interplay is thereby indicated between the first-line TB drugs exerting alterations to the host metabolome during therapy.

This is the first study of its kind to indicate changes to the host metabolome in response to intensive TB treatment, at different time intervals during the course of treatment. These results subsequently provide new insights into the mechanisms of TB drug metabolism, drug action, and drug-related side effects, thereby paving the way for the development of improved therapeutic approaches for the disease, and perhaps more importantly, also for monitoring treatment progression.

\section{Acknowledgments}

The authors thank Fanie Kamfer for assisting with the metabolite extraction, Derylize Beukes and Emile Jansen van Rensburg for their support with the GCxGC-TOFMS analyses, Mari van Reenen and Gooitzen Zwanenburg for assistance with the statistical data analyses and the ASCA analysis.

\section{Author Disclosure Statement}

The authors declare they have no conflicting financial interests.

\section{Funding Information}

The financial assistance of the National Research Foundation (NRF) of South Africa for this research is gratefully acknowledged (UID:117496). The opinions expressed and conclusions derived are those of the authors and are not necessarily those of the NRF.

\section{References}

Bando K, Kunimatsu T, Sakai J, et al. (2011). GC-MS-based metabolomics reveals mechanism of action for hydrazine induced hepatotoxicity in rats. J Appl Toxicol 31, 524-535.

Biswas SK, and Rahman I. (2009). Environmental toxicity, redox signaling and lung inflammation: The role of glutathione. Mol Aspects Med 30, 60-76.

Cao J, Mi Y, Shi C, et al. (2018). First-line anti-tuberculosis drugs induce hepatotoxicity: A novel mechanism based on a urinary metabolomics platform. Biochem Biophys Res Commun 497, 485-491.

Charles CJ, Rout SP, Garratt EJ, Patel K, Laws AP, and Humphreys PN. (2015). The enrichment of an alkaliphilic biofilm consortia capable of the anaerobic degradation of isosaccharinic acid from cellulosic materials incubated within an anthropogenic, hyperalkaline environment. FEMS Microbiol Ecol 91, 1-11.

Che NY, Cheng JH, Li HJ, et al. (2013). Decreased serum 5oxoproline in TB patients is associated with pathological damage of the lung. Clin Chim Acta 423, 5-9.

Das MK, Arya R, Debnath S, et al. (2016). Global urine metabolomics in patients treated with first-line tuberculosis drugs and identification of a novel metabolite of ethambutol. Antimicrob Agents Chemother 60, 2257-2264.

Das MK, Bishwal SC, Das A, et al. (2015). Deregulated tyrosinephenylalanine metabolism in pulmonary tuberculosis patients. J Proteome Res 14, 1947-1956.

Demartini B, Camisasca M, Zuin M, and Gambini O. (2013). A case of isoniazid-induced delirium. J Neuropsych Clin Neurosci 25, E68-E69.

De Villiers L, and Loots DT. (2013). Using metabolomics for elucidating the mechanisms related to tuberculosis treatment failure. Curr Metabolomics 1, 306-317.

Dierks T, Dickmanns A, Preusser-Kunze A, et al. (2005). Molecular basis for multiple sulfatase deficiency and mechanism for formylglycine generation of the human formylglycinegenerating enzyme. Cell 121, 541-552.

Du Preez I, and Loots DT. (2013). New sputum metabolite markers implicating adaptations of the host to Mycobacterium tuberculosis, and vice versa. Tuberculosis (Edinb) 93, 330-337.

Du Preez I, and Loots DT. (2018). Novel insights into the pharmacometabonomics of first-line tuberculosis drugs relating to metabolism, mechanism of action and drug-resistance. Drug Metab Rev 50, 466-481.

Du Preez I, Luies L, and Loots DT. (2019). The application of metabolomics toward pulmonary tuberculosis research. Tuberculosis 115, 126-139.

Everett JR, Loo RL, and Pullen FS. (2013). Pharmacometabonomics and personalized medicine. Ann Clin Bochem 50, 523-545.

Flint HJ, Scott KP, Duncan SH, Louis P, and Forano E. (2012). Microbial degradation of complex carbohydrates in the gut. Gut Microbes 3, 289-306.

Gatley SJ, and Sherratt HS. (1977). The synthesis of hippurate from benzoate and glycine by rat liver mitochondria. Submitochondrial localization and kinetics. Biochem J 166, 39-47.

Herold DA, Keil K, and Bruns DE. (1989). Oxidation of polyethylene glycols by alcohol dehydrogenase. Biochem Pharmacol 38, 73-76.

Holmes RP, and Assimos DG. (1998). Glyoxylate synthesis, and its modulation and influence on oxalate synthesis. J Urol 160, 1617-1624.

Hu Y, Yang Q, Liu B, et al. (2019). Gut microbiota associated with pulmonary tuberculosis and dysbiosis caused by antituberculosis drugs. J Infect 78, 317-322.

Jamaati H, Mortaz E, Pajouhi Z, et al. (2017). Nitric oxide in the pathogenesis and treatment of tuberculosis. Front Microbiol 8, 2008.

Knop M, Dang TQ, Jeschke G, and Seebeck FP. (2017). Copper is a cofactor of the formylglycine-generating enzyme. Chembiochem 18, 161-165.

Koen N, Du Preez I, and Loots DT. (2016). Chapter threeMetabolomics and personalized medicine. Adv Protein Chem Struct Biol 102, 53-78.

Kumar S, and Punekar NS. (1998). Inhibition of succinic semialdehyde dehydrogenase by $\mathrm{N}$-formylglycine. J Enzyme Inhib 13, 369-376. 
Lambert ML, Hasker E, Van Deun A, Roberfroid D, Boelaert M, and Van Der Stuyft P. (2003). Recurrence in tuberculosis: Relapse or reinfection? Lancet Infect Dis 3, 282-287.

Lamichhane G. (2011). Mycobacterium tuberculosis response to stress from reactive oxygen and nitrogen species. Front Microbiol 2, 176.

Lee SY, Jang H, Lee JY, Kwon KI, Oh SJ, and Kim SK. (2014). Inhibition of cytochrome $\mathrm{P} 450$ by ethambutol in human liver microsomes. Toxicol Lett 229, 33-40.

Lieber CS. (1997). Ethanol metabolism, cirrhosis and alcoholism. Clin Chim Acta 257, 59-84.

Loots DT, Wiid IJ, Page BJ, Mienie LJ, and Helden PD. (2005). Melatonin prevents the free radical and MADD metabolic profiles induced by antituberculosis drugs in an animal model. J Pineal Res 38, 100-106.

Luckner SR, Machutta CA, Tonge PJ, and Kisker C. (2009). Crystal structures of Mycobacterium tuberculosis KasA show mode of action within cell wall biosynthesis and its inhibition by thiolactomycin. Structure 17, 1004-1013.

Luies L, and Loots DT. (2016). Tuberculosis metabolomics reveals adaptations of man and microbe in order to outcompete and survive. Metabolomics 12, 40-49.

Mikusova K, Mikus M, Besra GS, Hancock I, and Brennan PJ. (1996). Biosynthesis of the linkage region of the mycobacterial cell wall. J Biol Chem 271, 7820-7828.

Mirsaeidi M, and Sadikot R. (2018). Patients at high risk of tuberculosis recurrence. Int J Mycobacteriol 7, 1-6.

Namasivayam S, Sher A, Glickman MS, and Wipperman MF. (2018). The microbiome and tuberculosis: Early evidence for cross talk. mBio 9, e01420-18.

Nanda GS, Sharma B, and Arora A. (2016). Adverse reactions due to directly observed treatment short course therapy: An Indian prospective study. IAIM 3, 6-12.

Ngwuluka N. (2010). Application of in situ polymerization for design and development of oral drug delivery systems. AAPS PharmSciTech 11, 1603-1611.

Nicholls AW, Holmes E, Lindon JC, et al. (2001). Metabonomic investigations into hydrazine toxicity in the rat. Chem Res Toxicol 14, 975-987.

Nicholson JK, Lindon JC, and Holmes E. (1999). 'Metabonomics': Understanding the metabolic responses of living systems to pathophysiological stimuli via multivariate statistical analysis of biological NMR spectroscopic data. Xenobiotica 29, 1181-1189.

Nicholson S, Bonecini-Almeida MDG, Lapa E Silva JR, et al. (1996). Inducible nitric oxide synthase in pulmonary alveolar macrophages from patients with tuberculosis. J Exp Med 183, 2293.

Niwa T, Maeda K, Ohki T, Saito A, Sakakibara J, and Kobayashi K. (1980). Gas chromatographic-mass spectrometric identification of isosaccharino-1,4-lactone in human serum and urine. Clin Chem 26, 1554-1561.

Olivier I, and Loots DT. (2012). A metabolomics approach to characterise and identify various Mycobacterium species. J Microbiol Methods 88, 419-426.

Peets EA, and Buyske DA. (1964). Comparative metabolism of ethambutol and its l-isomer. Biochem Pharmacol 13, 1403-1419.

Pelkonen O, Turpeinen M, Hakkola J, Honkakoski P, Hukkanen J, and Raunio H. (2008). Inhibition and induction of human cytochrome P450 enzymes: Current status. Arch Toxicol 82, 667-715.

Pole S, Maurya S, Hasnale P, Rathod N, Bendale S, and Khutle NM. (2016). A detail understanding of enteric coated tablet: Manufacturing and evaluation. Eur J Pharm Med Res 3, 135-144.

Ramappa V, and Aithal GP. (2013). Hepatotoxicity related to anti-tuberculosis drugs: Mechanisms and management. J Clin Exp Hepatol 3, 37-49.

Reinecke CJ, Koekemoer G, Van Der Westhuizen F, et al. (2012). Metabolomics of urinary organic acids in respiratory chain deficiencies in children. Metabolomics 8, 264-283.

Ronacher K, Chegou NN, Kleynhans L, et al. (2019). Distinct serum biosignatures are associated with different tuberculosis treatment outcomes. Tuberculosis 118, 101859.

Sarkar S, Ganguly A, and Sunwoo H. (2016). Current overview of anti-tuberculosis drugs: Metabolism and toxicities. Mycobact Dis 6, 1-6.

Sato T, Kusuhara S, Yokoi W, Ito M, and Miyazaki K. (2017). Prebiotic potential of L-sorbose and xylitol in promoting the growth and metabolic activity of specific butyrate-producing bacteria in human fecal culture. FEMS Microbiol Ecol 93, $1-10$.

Saukkonen JJ, Cohn DL, Jasmer RM, et al. (2006). An official ATS statement: Hepatotoxicity of antituberculosis therapy. Am J Respir Crit Care Med 174, 935-952.

Shastri MD, Shukla SD, Chong WC, et al. (2018). Role of oxidative stress in the pathology and management of human tuberculosis. Oxid Med Cell Longev 2018, 1-10.

Shibata K, Fukuwatari T, and Sugimoto E. (2001). Effects of dietary pyrazinamide, an antituberculosis agent, on the metabolism of tryptophan to niacin and of tryptophan to serotonin in rats. Biosci Biotechnol Biochem 65, 13391346.

Shinka T, Inoue Y, Ohse M, et al. (2002). Rapid and sensitive detection of urinary 4-hydroxybutyric acid and its related compounds by gas chromatography-mass spectrometry in a patient with succinic semialdehyde dehydrogenase deficiency. J Chromatogr B Analyt Technol Biomed Life Sci 776, 57-63.

Silhavy TJ, Kahne D, and Walker S. (2010). The bacterial cell envelope. CSH Perspect Biol 2, 1-16.

Smilde AK, Jansen JJ, Hoefsloot HC, Lamers RJ, Van Der Greef J, and Timmerman ME. (2005). ANOVA-simultaneous component analysis (ASCA): A new tool for analyzing designed metabolomics data. Bioinformatics 21, 3043-3048.

Smirnoff N. (2018). Ascorbic acid metabolism and functions: A comparison of plants and mammals. Free Radic Biol Med 122, 116-129.

Sodhi CP, Rana SV, Mehta SK, Vaiphei K, Attari S, and Mehta S. (1997). Study of oxidative-stress in isoniazid-rifampicin induced hepatic injury in young rats. Drug Chem Toxicol 20, 255-269.

Strolin Benedetti M, Whomsley R, and Baltes E. (2006). Involvement of enzymes other than CYPs in the oxidative metabolism of xenobiotics. Expert Opin Drug Metab Toxicol 2, 895-921.

Swart A, and Harris V. (2005). Drug interactions with tuberculosis therapy. Contin Med Educ 23, 56-60.

Tabachnick B, and Fidell L. (2007). Experimental Designs using ANOVA. Belmont: Thomson Brooks/Cole.

Takeda K, Watanabe J, Inoue K, and Kanamura S. (2000). Rifampicin suppresses hepatic CYP2E1 expression and minimizes DNA injury caused by carbon tetrachloride in perivenular hepatocytes of mice. Alcohol Clin Exp Res 24, 87s-92s.

Tretter L, and Adam-Vizi V. (2000). Inhibition of Krebs cycle enzymes by hydrogen peroxide: A key role of [alpha]- 
ketoglutarate dehydrogenase in limiting NADH production under oxidative stress. J Neurosc 20, 8972-8979.

Van Acker H, and Coenye T. (2017). The role of reactive oxygen species in antibiotic-mediated killing of bacteria. Trends Microbiol 25, 456-466.

Van Den Berg RA, Hoefsloot HCJ, Westerhuis JA, Smilde AK, and Van Der Werf MJ. (2006). Centering, scaling and trabsformations: Improving the biological information content of metabolomics data. BMC Genomics 7, 142.

Varma K. (2016). Excipients used in the formulation of tablets. Res Rev J Chem 5, 143-154.

Vilcheze C, Hartman T, Weinrick B, and Jacobs WR Jr. (2013). Mycobacterium tuberculosis is extraordinarily sensitive to killing by a vitamin C-induced Fenton reaction. Nat Commun 4, 1881.

Walder AD, and Tyler CK. (1994). Ethylene glycol antifreeze poisoning. Three case reports and a review of treatment. Anaesthesia 49, 964-967.

Watford M. (2003). The urea cycle: Teaching intermediary metabolism in a physiological setting. Biochem Mol Biol Educ 31, 289-297.

WHO. (2018). Global tuberculosis report 2018. Geneva, Switzerland: WHO Press, p. 272.

Würsch P, Welsch C, and Arnaud MJ. (1979). Metabolism of L-sorbose in the rat and the effect of the intestinal microflora on its utilization both in the rat and in the human. Ann Nutr Metab 23, 145-155.

Address correspondence to:

Dr. Ilse du Preez

Human Metabolomics North-West University

Potchefstroom Campus Private Bag x6001, Box 269 Potchefstroom 2531

South Africa

E-mail: ilse.dupreez@nwu.ac.za

\section{Abbreviations Used}

$\mathrm{ADH}=$ alcohol dehydrogenase

ANOVA $=$ analyses of variance

ASCA $=$ ANOVA simultaneous component analysis

BSTFA $=O$-bis(trimethylsilyl)trifluoroacetamide

$\mathrm{CYP}=$ cytochrome $\mathrm{P} 450$

DOTS $=$ directly observed treatment, short course

$\mathrm{EMB}=$ ethambutol

$\mathrm{FGE}=$ formylglycine-generating enzyme

GCxGC-TOFMS = two-dimensional gas chromatography time-of-flight mass spectrometry

$\mathrm{HCl}=$ hydrochloric acid

$\mathrm{HIV}=$ human immunodeficiency virus

$\mathrm{INH}=$ isoniazid

$\mathrm{LV}=$ levels of variance

M. tuberculosis $=$ Mycobacterium tuberculosis

$\mathrm{NAD} / \mathrm{NADH}=$ nicotinamide adenine dinucleotide

$\mathrm{Na}_{2} \mathrm{SO}_{4}=$ sodium sulfate anhydrous

$\mathrm{PCA}=$ principal component analysis

PEG = polyethylene glycol

POA $=$ pyrazinoic acid

$\mathrm{PZA}=$ pyrazinamide

$\mathrm{QC}=$ quality control

$\mathrm{RIF}=$ rifampicin

$\mathrm{RM}=$ repeated measures

ROS $=$ reactive oxygen species

SSADH $=$ succinic semialdehyde dehydrogenase

$\mathrm{SSL}=$ sum of the squared loadings

$\mathrm{TB}=$ tuberculosis

TCA $=$ tricarboxylic acid

TMCS $=$ trimethylchlorosilane

$\mathrm{WHO}=$ World Health Organization 